\title{
Time-Domain Analysis of RF and Microwave Autonomous Circuits by Vector Fitting-Based Approach
}

\author{
Leonardo Pantoli ${ }^{1 \#}$, Domenico Spina ${ }^{2}$, Daniele Romano ${ }^{1}$, Giulio Antonini ${ }^{1}$, Giorgio Leuzzi ${ }^{1}$, Tom Dhaene ${ }^{2}$ \\ ${ }^{1}$ Department of Industrial and Information Engineering and Economics, University of L'Aquila, \\ Monteluco di Roio, 67100, L'Aquila, Italy \\ ${ }^{2}$ IDLab, Department of Information Technology, Ghent University - imec, Technologiepark-Zwijnaarde 15, \\ B-9052 Ghent, Belgium \\ \#leonardo.pantoli@univaq.it
}

\begin{abstract}
This work presents a new method for the analysis of RF and microwave autonomous circuits directly in the time-domain, which is the most effective approach at simulation level to evaluate nonlinear phenomena. For RF and microwave autonomous circuits, time-domain simulations usually experiment convergence problems or numerical inaccuracies due to the presence of distributed elements, preventing de-facto their use. The proposed solution is based on the Vector Fitting algorithm applied directly at circuit level. A case study relative to a $\mathbf{R F}$ hybrid oscillator is presented for practical demonstration and evaluation of performance reliability of the proposed method.

Keywords - Autonomous circuits, microwave oscillators, radio frequency, time-domain, vector fitting.
\end{abstract}

\section{INTRODUCTION}

For circuit design, time-domain simulation is the primary solution at low frequency, since it is the more effective and general [1]. Unfortunately, this approach can be rarely applied at high frequency, where distributed elements and custom geometries often defined only at EM-level are employed, especially in monolithic circuits. In fact, usually transient analysis of RF and microwave circuits presents convergence problems or collapse to a trivial solution (null signal) in case of autonomous circuits. Therefore, frequency-domain analysis methods (such as Harmonic Balance) are well established for $\mathrm{RF}$ and microwave circuits design, and several techniques have been developed in the literature to obtain consistent results [2]. However, this approach exhibits also some drawbacks [3]: the analysis is restricted to periodic or quasi-periodic regime by definition, therefore excluding, for instance, the turn-on of an oscillator. In addition, a general limitation is relative to the analysis of potentially unstable circuits in which autonomous frequency components may arise, since they are not included a-priori in the analysis. To overcome this problem, specific methods based on data interpolation have been developed [4], [5], which assume that a spurious signal is present and that a rough estimation of its frequency is known.

Therefore, from a design point of view, the capability to perform transient simulations also on RF and microwave circuits could be very useful, especially when considering nonlinear or autonomous circuits. In fact, this will allow to verify with high accuracy both the start-up conditions and the evolution over time of the state of a nonlinear circuit, so avoiding the limitations given by frequency-domain based methods [4].

This work presents an innovative analysis method which allows one to simulate directly in the time-domain RF and microwave autonomous circuits. The proposed approach relies on the Vector Fitting (VF) algorithm [6], [7] to model the whole passive part of a nonlinear RF circuit, which is usually responsible of the lack of convergence when traditional time-simulation analysis methods are used at high frequency. In particular, first the transfer function of the linear and passive network of the nonlinear circuit considered is computed over a discrete set of frequencies over the band of interest, then a suitable model in a poles-residues form in the Laplace-domain is obtained by means of the VF algorithm [6]. Finally, such model can be converted into a corresponding equivalent circuit, composed only by lumped elements and controlled sources [8].

The proposed approach is general and, by properly combining the obtained equivalent model and the nonlinear active device(s), it becomes possible to simulate an autonomous circuit directly in the time-domain. VF has been widely used in the literature for different applications [9], [10]; however, in the framework of RF and microwave circuit simulations, the VF algorithm has been studied only at system level [11]. A suitable application example, based on a discrete oscillator operating in UHF band, is also presented and validates the proposed approach.

\section{AnAlysis Method}

General microwave networks (including autonomous circuits) are formed by an arbitrary number of nonlinear components and linear elements (distributed and/or lumped). Let us assume that the electromagnetic coupling between the distributed linear parts and nonlinear devices is sufficiently small to be considered negligible. Then, it is possible to model separately the linear and passive elements and the nonlinear devices: such models can then be suitably combined to describe the entire microwave network considered. In the following, we will assume that the models of the nonlinear elements are given (i.e. libraries provided by the foundry, 
behavioral models) and we will focus on the calculation of a suitable equivalent circuit for the linear and passive part of the autonomous circuit considered.

\section{A. Vector Fitting-based modeling}

Let us assume that the transfer function of the linear and passive system under study, which can be expressed in different forms (such as impedance, admittance and scattering parameters) has been estimated via full-wave EM simulations or measurements for a discrete set of frequency values in the bandwidth of interest for the application considered: $\mathbf{H}\left(f_{k}\right)$ for $f_{k}=1, \ldots, K$. The VF algorithm allows for computing a continuous pole-residue representation in the form [6], [7]

$$
\mathbf{H}(s)=\sum_{q=1}^{Q} \frac{\mathbf{R}_{q}}{s-p_{q}}+\mathbf{D},
$$

where $s=j 2 \pi f$ is the Laplace variable, $p_{q}$ are the poles, which can be either real or complex, and $\mathbf{R}_{q} \in C^{N \times N}$ are the corresponding residues, while $\mathbf{D} \in R^{N \times N}$ is a real matrix and $N$ is the number of input and output (I/O) ports of the system considered. Typically, all matrix elements of the transfer-matrix representation $\mathbf{H}(s)$ use a common pole-set $\left[p_{1}, p_{2}, \cdots, p_{n_{p}}\right]$.

Note that, fundamental properties for time-domain simulations such as the stability and passivity of models in the form (1) must be assured [9]. While stability can be guaranteed by construction by employing suitable pole flipping schemes [6], the pole-residue model (1) might be non passive, due to unavoidable numerical approximations in the model building phase. In this case, its passivity can be verified and enforced as a post processing step by means of dedicated passivity enforcement techniques [12], [13].

One of the main features of the pole-residue representation (1), apart from its ability to preserve the system physical properties (i.e. stability and passivity), is that it can be easily converted into an equivalent stable and passive state-space representation [6], which is suitable for time-domain simulations:

$$
\begin{aligned}
\frac{d}{d t} \mathbf{x}(t) & =\mathbf{A x}(t)+\mathbf{B u}(t) \\
\mathbf{y}(t) & =\mathbf{C x}(t)+\mathbf{D u}(t)
\end{aligned}
$$

where $\mathbf{A} \in R^{M \times M}, \mathbf{B} \in R^{M \times N}, \mathbf{C} \in R^{N \times M}, \mathbf{D} \in R^{N \times N}$ and $M$ is the order of the model, depending on the number of ports $N$ and poles $Q$.

\section{B. Circuit analysis}

State-space models are inherently systems of first-order ordinary differential equations (ODE): several of robust ODE solvers can be adopted to solve (2) with accuracy and efficiency. However, computer aided design (CAD) simulators such as SPICE do not directly accept differential equations as input. The representation (2) must be converted into an equivalent circuit and several techniques have been presented in the literature to reach this goal, such as [14], [15]. Note that such equivalent circuit is SPICE compatible, since it is formed only by lumped elements and controlled sources. Finally, the obtained circuit can be connected to the models of the nonlinear device(s) for time-domain simulations of the autonomous circuit considered.

The proposed methodology is flexible and it can be applied to any autonomous circuit. However, two possible elements can limit its application: if a large number of poles is needed in (1) to describe accurately the system frequency response, the computational cost of passivity enforcement schemes can become unfeasible for systems with a large number of I/O ports; the DC operating condition of the circuit must be accurately estimated, in order for the model to reproduce the conditions for the initial trigger of the autonomous behaviour of the circuit.

Note that the proposed modeling and simulation approach is more general than convolution-based methods: transient solvers with both fixed and adaptive time step can be used for time-domain simulations, since the model is continuous with respect to the frequency and time; while performance of convolution-based methods depend on the sampling strategy adopted in the frequency-domain to compute the system transfer function.

\section{APPLICATION EXAMPLE}

The proposed method has been applied to the design of an RF oscillator operating in UHF band. This is an hybrid prototype, based on the BFR92A, a medium-power BJT from Infineon, and conceived on a TLX8 substrate with microstrips and SMD passive components. The architecture of the oscillator is synthesized in Fig. 1a, which also shows the photograph of the oscillator prototype. In particular, a passive LC resonator is the input stage of the active section, realized with a negative resistance gain stage, while an output passive network is used to provide the start-up condition of the oscillator. Usually, autonomous circuits with distributed components cannot be simulated with accuracy in the time-domain, since convergence problems often occur. The solution here addressed overcomes this problem by analyzing the circuit as follows.

The passive networks of the entire circuit are considered as a single linear and passive system to be modeled, as shown in Fig. 1b at layout level. A 5x5 network has been defined since both DC and RF ports are included in the modelling and these are indicated in Fig. 1b by arrows. More in details, the following ports have been considered: RF output, base terminal, collector terminal, emitter terminals and DC bias pad. It is important to note that also the DC bias port is taken into account in order to analyze the circuit in a wide frequency range also considering the matching on the bias line. So, the netlist-based equivalent network is defined from DC up to high frequency in order to include all significant harmonics of the input signal and properly analyze the nonlinearities of the circuit.

By applying the VF algorithm, it is possible to choose the transfer function representation ( $\mathrm{S}-\mathrm{Y}-\mathrm{Z}$ parameters) more 


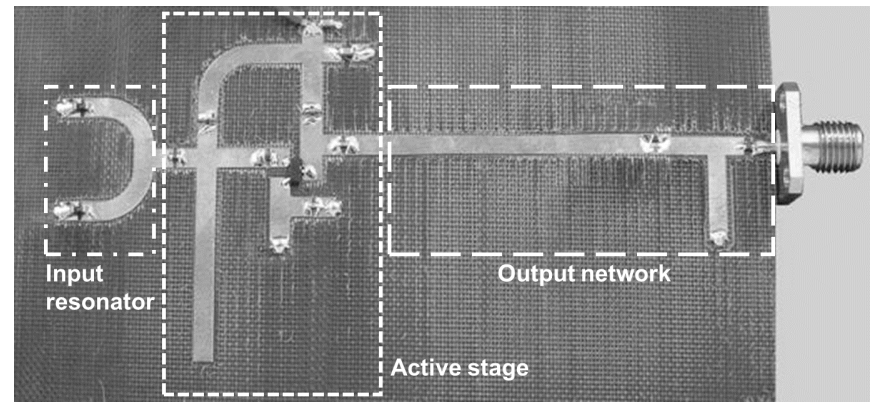

(a)

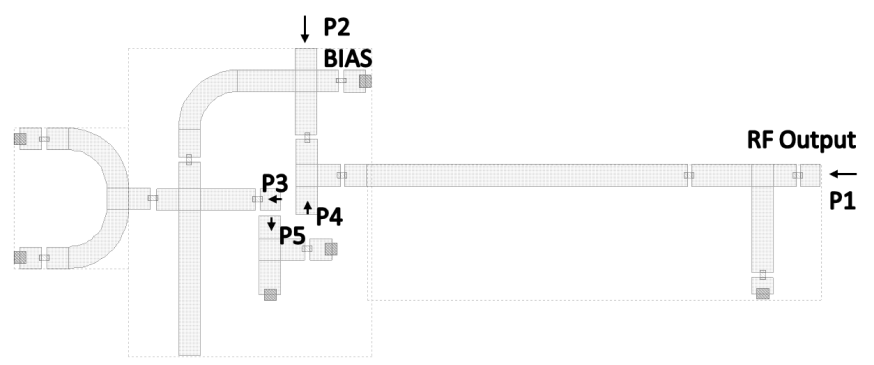

(b)

Fig. 1. Photograph of the oscillator prototype (a) together with its layout (b), whose linear and passive networks have been modeled by VF. I/O ports have been also pointed out.

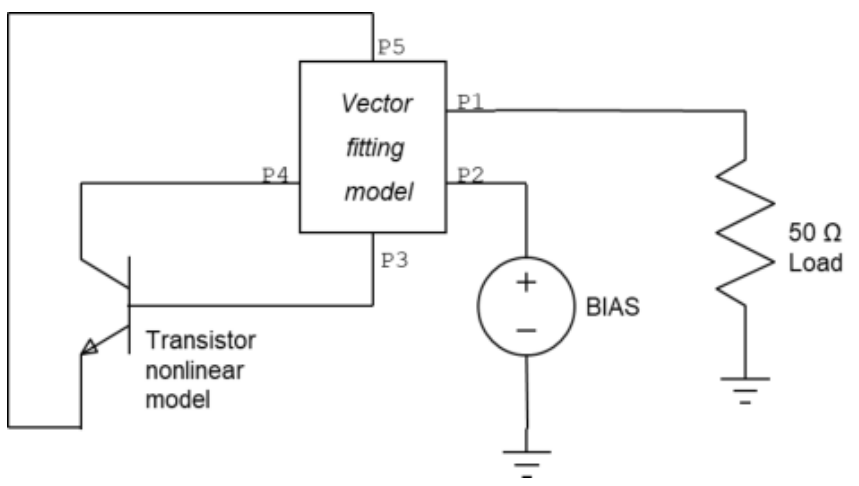

Fig. 2. Post modeling schematic simulating the hybrid oscillator. Both the nonlinear device and the passive network are now represented by netlist. The same ports numbers of Fig. $1 \mathrm{~b}$ are reported for correspondence.

suitable to the problem at hand, also optimizing the modeling parameters with respect to the data characteristics, the circuit topology and the CAD simulation software in use. In this example, the admittance parameters of the network considered have been computed via full-wave simulations in the frequency range [0 - 4.7] GHz. Next, a rational model with 49 poles in the form (1) has been obtained via the VF algorithm. A total of 941 linearly spaced frequency samples have been obtained, but only 641 have been used to compute a rational model in the form (1), the rest has been used to verify its accuracy in the entire frequency range considered, as shown in Fig. 3. This operation allows one to evaluate the modeling accuracy for samples not used to build the pole/residues representation. Finally, an equivalent circuit containing only lumped $R, L$ and $C$ elements has been obtained via the technique [14].

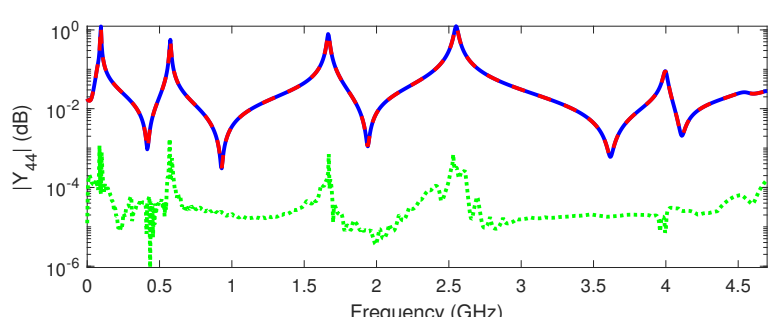

(a)

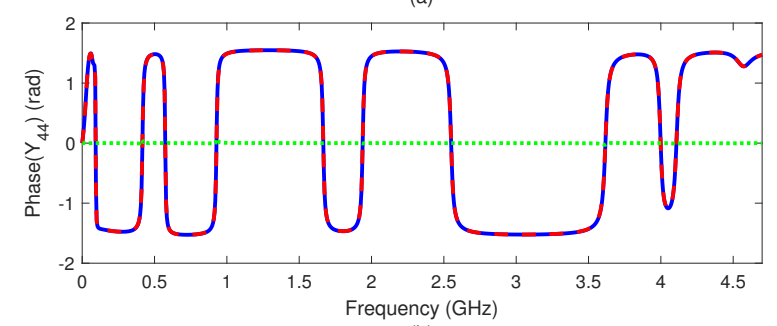

(b)

Fig. 3. Modeling results. (a): magnitude of the element $Y_{44}$ estimated via full-wave simulations (full blue line) and via the computed VF model (red dashed line) over 941 linearly spaced frequency samples. The magnitude of the corresponding absolute error is indicated by a green dotted line. (b): phase of $Y_{44}$. The same color code used for (a) is adopted here, where the green dashed line represents the absolute error between the full-wave simulations and the VF model.

The oscillator can now be simulated at schematic level by considering the equivalent $\mathrm{N}$-ports network describing the passive structures and the active device, connected as shown in Fig. 2. The transistor is described with a Gummel-Poon nonlinear model, while the passive networks through the defined equivalent circuit. Therefore, the oscillator can now be simulated, without lack of generality, directly in the time-domain with any SPICE-equivalent solver. Some simulation results obtained by using, for instance, the Aplac Transient solver available in NI AWR CAD software are shown in Fig. 4. In particular, the time-domain, steady-state waveform of the synthesized output signal of the proposed oscillator is reported in Fig. 4a, while the corresponding spectrum computed via Fast Fourier Transform (FFT) is shown in Fig. $4 \mathrm{~b}$, which confirms the existence of an autonomous signal at $433 \mathrm{MHz}$, along with its harmonics.

It is important to remark that, in principle, a similar spectrum could be obtained also with a frequency-domain analysis. However, especially for autonomous signals, a rough estimation of the oscillation frequency should be a-priori available [16], [17] or the use of particular probes is required, such as auxiliary generators as perturbation of the circuit [4]. On the contrary, the transient behaviour cannot be investigated with a frequency-domain analysis, while it can be easily analyzed with the proposed approach.

Finally, an experimental verification of the achieved performance is provided in Fig. 5, where the measured output spectrum of the prototype is reported. Compared to the results in Fig. 5, the fundamental frequency, the output power and the main harmonic level estimated via simulations are very close to the corresponding measured values, so demonstrating the 


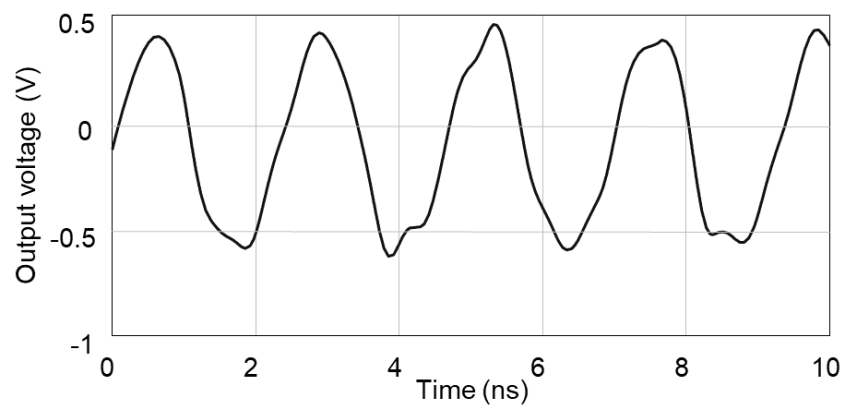

(a)

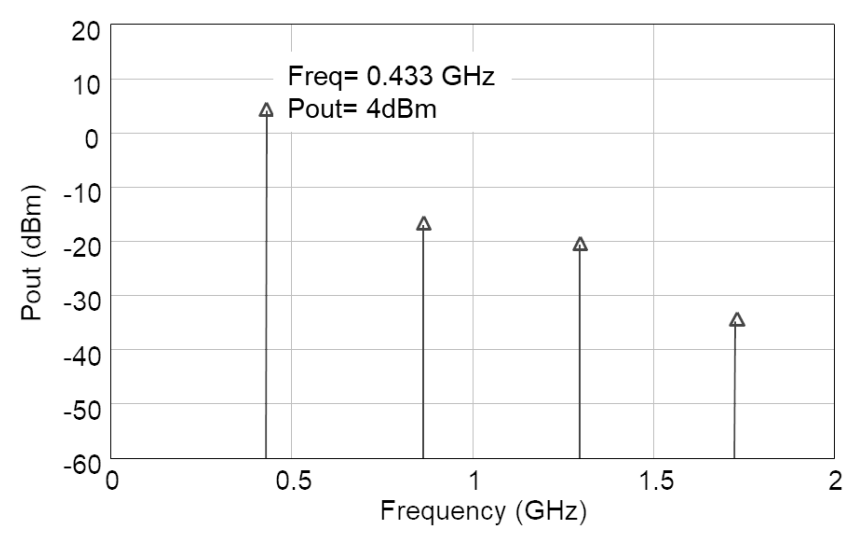

(b)

Fig. 4. Aplac Transient solver simulation results: (a) time-varying output signal of the proposed oscillator; (b) corresponding output spectrum computed via FFT.

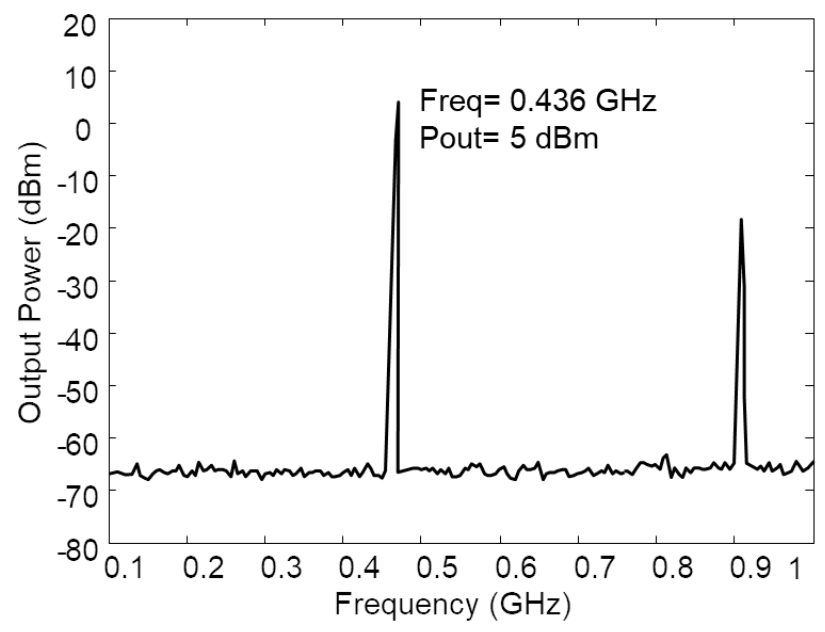

Fig. 5. Measured output spectrum of the hybrid oscillator.

feasibility of the proposed approach.

\section{CONCLUSION}

A novel method able to characterize directly in the time-domain RF and microwave autonomous circuits making use of distributed elements is presented in this work. Usually, autonomous circuits with distributed components cannot be simulated with accuracy in the time-domain, since convergence problems often occur. The proposed technique overcomes this limitation thanks to the modeling power of the VF algorithm, usually applied only at system level, and offers a great degree of flexibility in the modeling of the passive sections of the circuit under study. A relevant application example, the modeling and simulation of a discrete oscillator at UHF band, is presented and the obtained results are validated by measurements.

\section{REFERENCES}

[1] L. W. Nagel, "Spice2: A computer program to simulate semiconductor circuits," Ph.D. dissertation, EECS Department, University of California, Berkeley, 1975. [Online]. Available: http://www2.eecs.berkeley.edu/Pubs/TechRpts/1975/9602.html

[2] G. D. Vendelin, A. M. Pavio, and U. L. Rohde, Microwave Circuit Design Using Linear and Nonlinear Techniques. Wiley-IEEE Press, 2005.

[3] K. S. Kundert and A. Sangiovanni-Vincentelli, "Simulation of nonlinear circuits in the frequency domain," IEEE Transactions on Computer-Aided Design of Integrated Circuits and Systems, vol. 5, no. 4, pp. 521-535, October 1986.

[4] A. Suarez, Analysis and Design of Autonomous Microwave Circuits. Wiley-IEEE Press, 2009.

[5] L. Pantoli, "Transient-based conversion matrix approach for nonlinear stability analysis," Electronics Letters, vol. 50, no. 13, pp. 923-925, June 2014.

[6] B. Gustavsen and A. Semlyen, "Rational approximation of frequency domain responses by Vector Fitting," IEEE Trans. Power Del., vol. 14, no. 3, pp. 1052-1061, Jul. 1999.

[7] D. Deschrijver, M. Mrozowski, T. Dhaene, D. De Zutter, "Macromodeling of multiport systems using a fast implementation of the vector fitting method," IEEE Microw. Wirel. Compon. Lett., vol. 18, no. 6, pp. 383-385, Jun. 2008.

[8] R. Neumayer, F. Haslinger, A. Stelzer, and R. Weigel, "Synthesis of spice-compatible broadband electrical models from n-port scattering parameter data," in 2002 IEEE International Symposium on Electromagnetic Compatibility, vol. 1, Aug 2002, pp. 469-474 vol.1.

[9] Grivet-Talocia and Gustavsen, "Black-box macromodeling and its EMC applications," IEEE Electromagn. Compat., vol. 5, no. 3, pp. 71-78, Third 2016.

[10] D. Spina, F. Ferranti, G. Antonini, T. Dhaene, L. Knockaert and D. Vande Ginste, "Time-domain Green's function-based parametric sensitivity analysis of multiconductor transmission lines," IEEE Trans. Compon. Packag. Manuf. Technol., vol. 2, no. 9, pp. 1510-1517, Sept. 2012.

[11] T. J. Brazil, "Time-domain techniques in microwave circuit simulation," IET Microw. Antennas Propag., vol. 7, no. 2, pp. 146-155, Jan. 2013.

[12] B. Gustavsen, "Fast passivity enforcement for pole-residue models by perturbation of residue matrix eigenvalues," IEEE Trans. Power Del., vol. 23, no. 4, pp. 2278-2285, Oct. 2008.

[13] D. Deschrijver and T. Dhaene, "Fast passivity enforcement of $S$-parameter macromodels by pole perturbation," IEEE Trans. Microw. Theory Tech., vol. 57, no. 3, pp. 620-626, Mar. 2009.

[14] G. Antonini, "Spice compatible equivalent circuits of rational approximation of frequency domain responses," IEEE Electromagn. Compat., vol. 45, no. 3, pp. 502-512, Aug.

[15] J. F. Villena and L. M. Silveira, "Circuit synthesis for guaranteed positive sparse realization of passive state-space models," IEEE Trans. Circuits Syst. I, Reg. Papers, vol. 64, no. 6, pp. 1576-1587, Jun. 2017.

[16] L. Pantoli, G. Leuzzi, A. Santarelli, F. Filicori, and R. Giofrè, "Stabilisation approach for multi-device parallel power amplifiers under large-signal regime," in 2011 6th European Microwave Integrated Circuit Conference, Oct 2011, pp. 144-147.

[17] L. Pantoli, G. Leuzzi, A. Santarelli, and F. Filicori, "Stability analysis and design criteria of paralleled-device power amplifiers under large-signal regime," IEEE Transactions on Microwave Theory and Techniques, vol. 64 , no. 5 , pp. $1442-1455$, May 2016. 